\title{
6. Contemporary Physics and Discrete Aether
}

In this chapter I consider some theories that are prima facie relevant to the structure of the aether: String Theory, and more generally Supergravity theories; Loop Quantum Gravity; Causal Set theory; and Dynamical Triangulation.

String Theory, along with other Supergravity theories predicts particles that are yet to be discovered. Absent empirical confirmation, these theories, generated by the Consistent Renormalisation research program, as I call it, do not support a continuous aether theory. Rather they presuppose such a theory. On the other hand, Causal Set theory presupposes discrete aether, and its significance is largely that as usually formulated it presupposes Point Discretion. I shall argue that it becomes more plausible if adapted to Granulated Aether, and so has the same ontology as Dynamical Triangulation. As for Loop Quantum Gravity, the bad news is that in spite of appearances it offers no support for discrete theories nor does it disconfirm them.

There is not much joy, then, regarding the empirical evidence for or against discrete aether. I shall, however, obtain three results. The first, with which I begin the chapter, is that contemporary physics undermines an a priori argument against discrete theories of the aether, which I call the Argument from Scale Invariance. The second result is that discrete theories of the aether are threatened with a serious non-locality problem, and that Granulated Aether is superior to Point Discretion because of the way it deals with this threat. The third result shows that discrete aether is a high risk, high reward hypothesis, with symmetric continuous aether being low risk and low reward. That is because, I argue, discrete aether is committed to geometrodynamics, the thesis that all four fundamental forces are to be understood as a consequence of the detailed shape of the aether. ${ }^{19}$ By contrast, symmetric continuous aether requires the treatment of gravity as somewhat similar to other forces. The differences between the two ways of unifying physics are: 
1. The combination of Pseudo-set Granules, as the preferred version of Granulated Aether, with geometrodynamics promises a theory that is fundamentally mereological, with all other structure depending upon the mereology. There is also the hope of simple laws expressible in terms of positive integers. The result would be a triumph for simplicity. (That is the high reward). It would require a great deal of mathematics to relate this underlying structure to physics as we now have it, and it would be rash to assume this mathematics even exists (whether or not mathematicians discover it).

2. Treating gravity much like any other force is straightforward in principle, and there are interpretations of quantum field theory that ensure consistency. I anticipate that the resulting unification will require several parameters to be adjusted to fit the empirical data, and so, if it is successful, it will not be as strikingly confirmed as discrete geometrodynamics would be.

Readers may not agree with my overall assessment that discrete geometrodynamics is less probable precisely because it is bolder. ${ }^{120}$ So my chief conclusion is disjunctive: The aether is either granulated, in which case probably Pseudo-set Granules and geometrodynamics are correct and simplicity triumphs, or the aether is symmetric Arntzenius Continuum, the hypothesis to be developed in the next chapter.

\section{Undermining the Argument from Scale Invariance}

Theoretical physicists rely heavily on the metaphysical principle that necessities should be highly symmetric. Thus the thesis that the shape of Space-time might have asymmetric geometry - Einstein likened it to the surface of a potato - is tied to the thesis that the general relativistic metric is (nomologically) contingent, and that the necessary structure of Space-time is merely its structure as a differentiable manifold, from which is derived the requirement that the laws of nature be covariant,

Karl Popper praised bold conjectures. He was also an avowed sceptic about ordinary induction. Those of us who are not sceptics could argue that bold conjectures are confirmed surprisingly often and so are not as improbable as we would judge a priori. 
that is statable in terms of the differentiable structure. This is a strong symmetry condition. Alternatively, if we require a necessary geometry such as that of Minkowski or de Sitter Space-time, then the requirements of isotropy (no privileged direction) and homogeneity (no privileged position) amount to symmetry conditions on the physically necessary.

One candidate symmetry is scale invariance, the thesis that, if all spatio-temporal distances were multiplied by a scale factor, this would leave the necessary structure of the universe unchanged. Taking the gravitational constant to be fixed, the empirical equivalence of our universe to one like ours but uniformly scaled up by a factor $\mu$ may be achieved only if there is also an accompanying multiplication of mass by $\mu$. So when I consider re-scaling I assume spatio-temporal distance and mass are both multiplied by some positive $\mu$.

The case for such scale invariance is that asymmetries are not to be multiplied more than is necessary. This is not an especially strong case, so it is worth noting the spurious empiricist argument for scale invariance that might have seemed to buttress it. If everything, including we ourselves, was scaled up or down, who could notice the difference? ${ }^{121}$ This rhetorical question presupposes that the aether is not discrete. For if it is discrete we may use the number of aether atoms as an absolute measure of quantity and scaling observers down so that they are of the scale of an aether-atom would be noticeable if, per impossibile, such scaled-down observers could exist. Therefore, it should not be used to argue against discrete aether.

Granulated Aether and Point Discretion do not violate scale invariance but rather make it vacuously true. For, absent unnecessary complications, they both imply an absolute measure of quantity and hence prohibit any change of scale. Such trivialisation is, however, contrary to the a priori appeal of scale invariance. I conclude that, unless undermined, the Argument from Scale Invariance provides a case against discrete theories, although not an especially strong one.

The way to undermine the argument is to show that scale invariance should be abandoned even on continuous aether hypotheses. For

121 The topic was discussed by, for instance by Schlesinger (1964) and Grünbaum (1964, 1967). 
instance the cosmological constant, $\Lambda$, would be affected by a change of scale unless it is exactly zero, contrary to the current consensus. The problem with this way of undermining the Argument from Scale Invariance is that $\Lambda$ might be considered nomologically contingent, and the consequence of the conditions early on in our part of the universe. There are many other examples of important physical constants that would have been different given a change of scale. Among these are the coupling constants that compare the strengths of various fields, for instance the fine-structure constant, $\alpha$. It is to be hoped that all these will be derived from a 'theory of everything'. This forces us to speculate about whether an as yet undiscovered theory will be scale-invariant. What we should expect depends on how firm is our intuition that symmetry should be maximised. Given a firm intuition we should expect a scaleinvariant theory of everything, but if the intuition is less firm we should be agnostic on that topic. Hence the argument from scale invariance against discrete theories of the aether merely supports my disjunctive conclusion, namely that if you back symmetry over simplicity you should hold the aether to be continuous. Otherwise, there is no case for scale invariance, and hence no obstacle to discrete theories of the aether.

\section{The need to derive Planck scale discrete aether}

If Granulated Aether is correct we might expect the hypervolume of a granule to be about 1 Planck unit, in which case their density in Planck units will be some constant $\rho$, which is of the order of magnitude 1 Planck unit, that is about 10173 granules per sec4. If Point Discretion holds then the aether atoms have zero hypervolume but we might again expect a density $\rho$ of about 10173 per sec 4 .

We should accept this estimate for the density of aether atoms given a discrete theory. For, I say, we should minimize the number of fundamental, that is, non-derived, physical constants - the stuff of fine tuning. To be sure, current physics is plagued with a proliferation of physical constants but the hope is that all or most of these are not genuine constants and vary from domain to domain of the universe. Moreover, humble metaphysicians that we are, we will be guided by physicists and take $\mathrm{G}, \mathrm{c}$ and $\mathrm{h}$ to be non-contingent. Hence the density of aether atoms 
$\rho$ would be a physical constant. It is not plausible that $\rho$ vary from domain to domain. In fact so intuitive is it that the hypervolume of a granule is a natural unit that if $\rho$ 's expression in Planck units varied, then we would take that as implying that one of $\mathrm{c}, \mathrm{G}$ or $\mathrm{h}$ varied, something we are here denying. Hence the only way to avoid $\rho$ being an unwanted fundamental physical constant is to derive it from the overall theory. An example of such a derivation is provided by the discrete interpretation of Loop Quantum Gravity, discussed below which, suitably interpreted, implies that the minimum area of a loop is $4 \log _{\mathrm{e}} 3$ (approximately 4.4) Planck units. (Baez 2003). How we interpret that minimum area will depend on both the shape of the aether granules and the local curvature of Space-time, but it is plausible that it is of the order of magnitude of a Planck unit, and, more important, determined by the structure of the aether.

So why should we be reluctant to admit non-derived physical constants? Because theoretical understanding is a matter of explaining the more complicated in terms of the less complicated. The number of nonderived constants is a partial measure of the complexity of a hypothesis and so, other things being equal, should be minimized.

Could we use the fine-tuning required for sentient life to undermine the case against the non-derived density of aether atoms? ${ }^{122}$ Not if we reject, as I have done, the variability of $\rho$ in a given universe. For if $\rho$ has a precise value, that it has this value rather than some other, almost equal, value (also suited to life) should be derived from the rest of the theory.

I conclude, then, that if the aether is discrete, $\rho$, the density of atoms per Planck hypervolume, should be predicted by the theory.

122 There is an extensive literature on fine-tuning in the philosophy of religion. (See Ratzsch 2012). As a case for the existence of God I prefer Coarse Tuning (Forrest 2007). 


\section{A challenge for discrete theories}

In the previous section I argued that a satisfactory quantum gravity based on discrete aether should be able to derive the density of atoms. There are two extreme cases. In the first we take a theory that does not support the discrete character of the aether, such as String Theory, and we nonetheless hypothesise that the aether is discrete. In that case, we are adding $\rho$ to the list of non-derived constants, which is a disadvantage compared to the case of continuous aether in which: (1) the most natural units are Planck units, so none of $\mathrm{G}, \mathrm{c}$ and $\mathrm{h}$ count as non-derived physical constants; and (2) the density of atoms $\rho$ is the only value it could have for a continuous theory, namely infinity.

There would be an even more serious disadvantage if the theory required structure at a scale much less than the Planck units. For instance, if String Theory required the strings to be of the order of a Planck unit in length, then to be strings and to vibrate we might hypothesise sub-Planck scale structure. And that would prevent the identification of the natural units with the Planck units. String Theory is, however, quite compatible with the strings typically being about 100 Planck units in length. ${ }^{123}$ We might then take the strings and branes to be one Planck unit thick. ${ }^{124}$ I conclude that if String Theory turns out to be correct the case against discrete aether, although of some weight, is far from conclusive.

The other extreme case is that in which taking the aether to be discrete we can derive an upper bound for $\rho$. For in that case, the discrete character of the aether explains why $h \neq 0$ and hence why classical theories are incorrect, something presupposed but not explained by the use of

123 At one stage it was assumed the strings would be of Planck scale length but later it was realised they would be a couple of orders of magnitude longer than that.

Even if the strings were a Planck unit in length there might be a way out, namely take the strings, branes etc to be specified by patterns of aether atoms rather than consisting of them. Consider, for instance aether atoms laid in a helical pattern around an axis in Space-time. Then the axis is distinguished without being made up of aether. 
Planck units. This would be a significant advantage for discrete theories of the aether.

\section{Against String Theory and Super-gravity.}

The strings and branes of String Theory are thin, but as far as I know there is no way of deriving their thickness or even of showing that they have non-zero thickness. Hence the argument of the previous section shows that theory to be somewhat improbable given discrete aether. The same holds for other Supergravity theories. So we have a case for not combining these theories with discrete aether. Maybe the disjunction of String theory and other Supergravity theories will be confirmed by the discovery of the novel particles they predict, such as gravitinos. That would, therefore, provide a case for continuous and hence symmetric aether, and also for Arntzenius Continuum. I now argue, however, that absent such empirical confirmation, String Theory and Supergravity are improbable - even relative to the hypothesis of continuous aether. My argument is that they are based on a research program the core of which presupposes (1) that the aether is continuous and (2) that there are no particles that are extended in all dimensions (i.e. of the same number of spatial dimensions as Space itself) Given the complexity of String Theory and of Supergravity more generally we should, I now argue, abandon this core, unless some empirical predictions are confirmed.

String Theory and other Supergravity theories belong to what I call the Consistent Renormalisation research program, which splendidly illustrates Imre Lakatos' methodology (Lakatos 1970). The core is the requirement that the standard approach to quantum field theory can be developed without divergent (i.e. infinite) integrals. This is in response to the unsatisfactory character of arbitrary and ad hoc cut-offs by which the energy and momenta of particles are bounded. (For instance to avoid the 'ultraviolet catastrophe' the cut-off ensures that the frequencies are less than some arbitrarily chosen very high value.) String Theory started with the brilliant idea that we might use strings to provide a theory of quarks and the associated strong nuclear force without cut-offs. It turned out that strings were not needed for that purpose, but meanwhile an anomaly was discovered, namely that String Theory predicted that some closed strings (i.e. ones closed into a loop and so without free ends) would have 
as excitations massless spin 2 particles, something not required for quark theory. This anomaly was then inverted into a 'prediction', by noting that gravitons (quantized gravitational waves) would be just such massless spin 2 particles. Consequently String Theory re-established itself as a theory of everything, that is, a unification of the theories of the electromagnetic, weak strong and gravitational forces. Another anomaly resulted from the discovery that there were five different string theories that could be converted into each other using transformations, known as dualities. (This is reminiscent of the notorious wave/particle duality, said to have been resolved by quantum field theory.) To resolve the duality, that is remove the anomaly, it was proposed that there must be a more basic underlying theory, which is known as M-theory and which posits branes, higher dimensional sheets to which the open strings are attached. This M-theory has now produced another anomaly, namely the proliferation of landscapes, that is models for the 'vacuum', that is, the matterfree universe. There are estimated to be about 10500 of these landscapes, in part because of the proliferation of the 6 dimensional manifolds describing the dimensions additional to the experienced 4 dimensions of Space-time. ${ }^{125}$ Some hope that of these landscapes only one will be suited to life.

As is well known, String Theory requires 6 extra dimensions. It is also a Super-gravity theory, requiring super-symmetry, a consequence of which is that the familiar particles (bosons and fermions) have superpartners (fermions and bosons respectively). For instance not only are gravitons implied by the theory but also gravitinos, fermions of spin $3 / 2$. Some of these super-partners are predicted to be discovered using the Large Hadron Accelerator. If they are not found, then this will be a further anomaly and it remains to see whether the research program could invert it into some further startling prediction.

125 These are vacuum solutions of the higher dimensional analog of General Relativity. Being massless they are 'Ricci flat'. They must also be compact. This forces on them a unique (differential) topology, that of a torus in 6 dimensions, but there is still an enormous proliferation of general relativistic metrics. See (Douglas and Kachru, 2007). 
The obvious criticism is that String Theory and Supergravity are just too complicated to be a fundamental theory, which as a 'theory of everything' it would have to be. This criticism may be developed into what I call the Modeling Objection, namely that a sufficiently rich and interesting mathematical structure has the resources to model an enormous range of fairly simple theories, but its success in so doing does not support the hypothesis that these are more than models. For instance a Quinean philosopher might claim that the fundamental structure of physics is that of set theory. And to be sure we can almost certainly model the correct physics set-theoretically if we can model it using mathematics. But that is a cheat, precisely because set theory is an almost universal model for mathematics. Likewise my complaint that String Theory is complicated may be developed into the argument that ten dimensional geometry is just so rich we expect it to model an enormous variety of theories, but that this too is cheating. In Lakatosian terms, I say that the research program degenerated as soon as the excessively rich 10 dimensional geometry was invoked, although the researchers were not aware of this richness at the time.

Here I distinguish two rather different appeals to simplicity. Even theories that are not fundamental should be relatively simple, that is as simple as we can make them given the empirical constraints, but for a fundamental theory we expect an absolutely simple theory. Or at least that is my metaphysical intuition, which I hope readers share. Chemistry in the year 1900, for example, seemed as simple as the evidence allowed but, because of the proliferation of chemical atoms, not simple enough to treated as fundamental, even at the time.

I have appealed to a metaphysical intuition concerning simplicity, but intuitions are defeasible and, I take it, proponents of String Theory consider that the intuition is defeated by the sheer difficulty of providing a consistent quantum field theory that would count as a 'theory of everything'. The history of the research program is one of force majeure: twists and turns required for consistency. When I say 'required' for consistency, I mean 'required to avoid other complexities'. For, as Jack Smart is fond of saying, any theory can be rendered consistent with enough ad hoc qualifications. 
The underlying principle is correct: if respect for the mathematics and the empirical constraints force complexity upon us then we are wrong in assuming the theory of everything must be simple. But this principle requires an extraordinary thoroughness if it is to be applied correctly. For it may happen that because of the accumulated complexities we should reconsider one of the earlier stages in the program.

There is a $10-33 \mathrm{~cm}$ elephant in the room - or is it a 20,000 nanogram gorilla? Discrete theories of the aether effortlessly prevent the ultraviolet catastrophe by putting an upper bound on frequencies of 1045 sec-1, the frequency of a gamma ray whose wavelength is $10-33 \mathrm{~cm}$ and whose energy is equivalent to 20,000 nanograms. Even if the aether is continuous, the ultraviolet catastrophe may be avoided by positing that the fundamental particles are extended regions of the aether with suitable properties - presumably their diameters will be of the order of magnitude of the Planck length. Their extended character forces upon us an upper bound to the particle density and hence the ultraviolet catastrophe is avoided. ${ }^{126}$ If there is an infrared catastrophe, then we can avoid that by resort to a spatially finite Space-time. The Consistent Renormalisation research program turns out to be based on a core of conservatism, with a reliance upon point particles. Initially that was sound methodology, but the excessive richness of 10 dimensional geometry has shown it has degenerated.

Nonetheless, I wish it well as a fun-filled research program, and if the prediction of super-particles is confirmed then the fact that these are implied by String Theory and Super Gravity, but not rivals, would have confirmed them. A corollary is that if they turn out, improbably, to be thus confirmed, then we should not combine them with discrete aether because the implications of String Theory are based on the twists and turns of a research program that presupposes the aether is continuous.

126 Compare the ideal gas law PV $=\mathrm{kT}$ (Pressure times volume equals a constant $\mathrm{k}$ times temperature). Even if we ignore the forces between molecules we need to make a correction to allow for the molecules not being point particles obtaining $\mathrm{P}(\mathrm{V}-\mathrm{b})=\mathrm{kT}$ where $\mathrm{b}$ is the sum of the volumes of the molecules themselves. 
For without continuity, the ultraviolet catastrophe would never have occurred in the first place.

\section{Does Loop Quantum Gravity imply discrete aether?}

Loop Quantum Gravity is based on the hypothesis that the correct quantum gravity will result from the (canonical) quantisation of General Relativity. It is cautious in that it is not intended as a 'theory of everything', and such caution increases its probability. Just how probable it is depends on the plausibility of canonical quantisation. The least kind judgement on canonical quantisation is that it is a recipe for coming up with one out of, for all we know, many possible theories that have the required classical limit in contexts in which Planck's constant may be ignored. To this can be added the admittedly weak support given by ordinary induction: canonical quantisation has worked fairly well in the cases of electrodynamics and chromodynamics (quark theory).

My interest in Loop Quantum Gravity derives from the way it implies a discrete spectrum for area and volume, a result that suggests the aether is discrete, even though the theory being quantised is not discrete. I say 'suggests' for just what a spectrum of an 'observable' is requires further, and contentious, interpretation. If we follow that suggestion we may argue by reductio ad absurdum. Within the scope of the supposition that the aether is continuous, Loop Quantum Gravity would arise, it is said, as a quantisation, which then would imply that the aether is not, after all, continuous. I shall argue, however, that the suggestion should be ignored.

Loop Quantum Gravity is motivated by the difficulty in quantising the way the aether curves. The difficulty is that quantum theory requires a volume-analog assignable to sets of the states in the configuration space, which in this case consists of all the physically possible ways the aether could be curved at a given time-coordinate $t$. (It is to be hoped that resulting quantum gravity does not depend on the choice of the $t$ coordinate.)

In the case of a single particle the configuration space is Space itself and the volume-analog the volume itself. Then the pure states correspond to the Hilbert space of square-integrable complex functions on the configuration space. It is easy to find volume-analogs for a given finite 
number of particles and then by considering more and more particles consider the case of any finite number of particles. The resulting Hilbert space is Fock space, used for instance in quantum electrodynamics. But how can we find a volume-analog on the configuration space for General Relativity?

Loop Quantum Gravity derives from a way of characterising the gravitational field due to Abhay Ashtekar, Giorgio Immirzi and Fernando Barbero, which enables us to find an appropriate volume-analog (Thiemann 2003: I.22). The idea is that curvature may be characterised by the way in which a quadruple of independent directions varies as it is transported around a closed loop. This is analogous to noting what happens to a pair of directions at, say, the South Pole if it is transported around a loop that goes up longitude zero to the equator, around to longitude 90 and then down again to the South Pole. It ends up as a different pair of directions because of the Earth's curvature. The transformation of the directions on the Earth's surface is given by a rotation, In the case of General Relativity the transformation is specified by a Lorentz transformation.

If we consider a family of loops joined up to form a graph with a finite number of vertices, the way the axes transform as we go round the graph gives us more information about the curvature the more loops we add in a given region. By considering a graph with a countable infinity of vertices in a given region the transformation associated with loops connecting any finite number of them can specify the curvature completely. Suppose we now take a set of four coordinate axes for a trip around the loop. Because of the curvature the overall result is that the axes undergo a transformation. At the cost of some arbitrariness (gauge freedom) we may analyse these transformations resulting from going around loops using Lorentz transformations as we take the axes from vertex to vertex. The result is that we may specify the general relativistic metric using a graph with countably many vertices, and hence countably many edges, assigning to each edge a Lorentz transformation. ${ }^{127}$ Hence

More generally, if we allow a connection with torsion - needed for taking intrinsic angular momentum (spin) into account - then the Lorentz group is replaced by the larger Poincaré group. This is important because it has SU(3) 
the metric for General Relativity is described by assigning a Lorentz transformation to each of countably many edges. As I understand it, Loop Quantum Gravity is the result of 'quantising' the system by assigning an irreducible representation of the Lorentz group to each edge. ${ }^{128}$ This procedure is analogous to the quantisation of electrodynamics where the quantum system is described using a countable infinity of one particle states. The chief difference is that we replace particles by edges.

In the resulting theory both the area of a loop and its volume have discrete spectra with minimum values obtained at the Planck scale. The precise values depend on the details of the theory. This result is suggestive of discrete aether, but merely suggestive, for the spectrum is not defined as the set of possible values but rather the set of eigenvalues. To obtain more than a mere suggestion, we would need some extra premise, such as the following Definite Range principle:

The value of an 'observable' is definitely in the range from the greatest lower bound to the least upper bound of the eigenvalues. Whether that principle holds depends on which quantities are given the status of 'observables'. But the case of spin shows that we should not grant that status too readily. For consider the values $\mathrm{S}_{\mathrm{X}}, \mathrm{S}_{\mathrm{y}}$ and $\mathrm{S}_{\mathrm{Z}}$ of the $\mathrm{x}, \mathrm{y}$ and $\mathrm{z}$ component of the spin of a spin $1 / 2$ particle. And suppose we grant observable status to their squares $\mathrm{S}_{\mathrm{x}}{ }^{2}, \mathrm{~S}_{\mathrm{y}}{ }^{2}$, and $\mathrm{S}_{\mathrm{z}}{ }^{2}$. These quantities have only one eigenvalue, $(\mathrm{h} / 4 \pi)^{2}$, so by the definite range principle all would definitely have that value, showing that $S_{X}= \pm(h / 4 \pi), S_{y}= \pm$ $(h / 4 \pi)$, and $S_{Z}= \pm(h / 4 \pi)$. So the spin-vector points from the centre of a cube of side $h / 2 \pi$ with edges parallel to the three axes, to one of the eight corners. This is absurd, because the choice of $\mathrm{x}, \mathrm{y}$ and $\mathrm{z}$ coordinates was arbitrary. What has gone wrong? A quibble is that the spin of a spin $1 / 2$

as a finite dimensional representation, and SU(3) is the symmetry group for unified strong/electro-weak theory.

The representation is by means of operators on the one-dimensional subspaces of a Hilbert space and hence corresponds to a representation by means of unitary operators of the covering group SU(2). These irreducible representations correspond to the spin states of particles of spin 0 (trivial), $1 / 2,1$ etc. 
particle is not really a vector, but we could consider instead a transversely polarised spin 1 boson with mass (i.e. not the photon) and its two transverse polarisations. But it is convenient to consider the more familiar example of spin 1/2. Either we should reject the Definite Range principle, or we should not assign observable status to components of spin, or we should deny that the square of an observable is always an observable. A similar argument would hold if we considered instead of $\mathrm{S}_{\mathrm{X}} 2$ $\mathrm{S}_{\mathrm{y}}{ }^{2}$, and $\mathrm{S}_{\mathrm{z}}{ }^{2}$ the magnitudes of the spin components: $\left|\mathrm{S}_{\mathrm{X}}\right|$, $\left|\mathrm{S}_{\mathrm{y}}\right|$, and $\left|\mathrm{S}_{\mathrm{z}}\right|$. So we would also have to deny that the magnitudes are observables.

We may now apply these three ways of dealing with the problem raised by spin to the case of area and volume. First, if we just deny the Definite Range principle the argument that there is a minimum area (and volume) collapses. Next, if we deny that quantities as physically important as the components of spin are observables then what right have we to assume area and volume are observables? Finally, if we deny that squares or absolute values of observables are observables, then I note that the area of a small parallelogram, or the volume of a small parallelepiped, may sensibly be interpreted as the scalar magnitudes (i.e. lengths) of the exterior products of two, or three respectively, of the vectors with length and direction of the sides, or edges respectively. Even if these exterior products (also known as antisymmetric tensors) are genuine observables then we have no reason to expect their magnitudes to be observables also. The situation may be illustrated by case of the area of a surface in three dimensions, in which case the exterior product may be replaced by the more familiar vector product. If we take a small parallelogram on the surface whose sides are the vectors $\mathbf{d s}$ and $\mathbf{d s} \mathbf{s}^{\prime}$ then their vector product $\mathbf{d s} \times \mathbf{d} \mathbf{s}^{\prime}$ is a vector perpendicular to the surface with magnitude equal to the area of the parallelogram. But $\mathbf{d s} \times \mathbf{d} \mathbf{s}^{\prime}=-\mathbf{d s} \mathbf{s}^{\prime} \times \mathbf{d s}$. That is, they are equal and opposite vectors. To calculate the surface area we do not integrate $\mathbf{d s} \times \mathbf{d} \mathbf{d} \mathbf{s}^{\prime}$, which for any finite surface without an edge would give us zero, because the vector products in one direction are balanced by those in the opposite direction. Instead, we have to integrate the magnitude of $\mathbf{d s} \times \mathbf{d} \mathbf{s}^{\prime}$.

The combination of continuous aether with Loop Quantum Gravity can be defended, therefore, even if the failure of the Definite Range 
principle is restricted to the magnitudes of signed or vector quantities that are genuine observables.

Loop Quantum Gravity offers, then, little support for discrete aether. Moreover, there is an argument to show that if treated as a fundamental theory of the aether it requires continuity. For it tells us that if we go round a loop, then the effect of the curvature is specified by a Lorentz transformation. So there must be something with the right structure for the Lorentz transformation to transform. If the cells were simples, however, then the corresponding transformation would be just a permutation of its vertices. If the permutation preserves orientation then it would be a member of the group A5, with 60 members. Maybe Loop Quantum Gravity could be modified so that this finite group replaces the Lorentz group. But that would not be Loop Quantum Gravity itself. As it is, Loop Quantum Gravity is more appropriate for a hypothesis in which the aether is made up of cells, each of which is flat and has the structure of a convex region of Minkowski Space-time. For in that case travelling around a loop would result in a Lorentz transformation.

This flat cell interpretation would not affect the entropy calculation for a black hole, which is an important 'prediction' of Loop Quantum Gravity (Ashtekar, Baez, Corichi and Krasnov 1998). For the flat internal structure of the cells would contribute zero entropy. Nor, it should be noted, does this settle the simplicity versus symmetry issue. For if we treat gravity as a field on a symmetric aether such as one with the structure of Minkowski Space-time then such a cell structure corresponds to a field restricted to the boundaries of the cells. I doubt, though, that we need draw the conclusion that there really is a cell structure for we may well think that quantum states with precise eigenvalues are idealisations and that actual states are always somewhat fuzzy being superpositions of such states. This would blur the sharpness of the boundaries.

Although there is no conclusive objection to combining Loop Quantum Gravity with a theory of the aether as consisting of cells each of which has the structure of a convex portion of Minkowski Spacetime, that hypothesis is not especially simple. For like granulation it requires some Planck level structure, but it does not have the advantage of discrete theories such as Pseudo-set Granules. So in the absence of greater empirical support we should reject it. 


\section{Causal Set theory}

Initially my preferred hypothesis was Point Discretion. In the previous chapter I noted two advantages that Granulated Aether might have over continuous aether in the case in which the aether is not symmetric, namely that of characterising a differentiable manifold. Whether Point Discretion shares these advantages depends on whether a certain hoped for result, which Sorkin calls a Hauptvermutung, can be proven. In addition there is the (temporal) Non-Locality Problem, arising from the nonlocality of the discrete analog of Minkowski Space-time. But first I shall sketch Causal Set theory to provide some context.

Causal Set theory, developed by Peter Szekeres (1995), Rafael Sorkin (2003) and others, presupposes Point Discretion. It is based on points with a single basic relation - one of the (interderivable) relations of absolute priority $(\mathrm{u} \prec \mathrm{v})$, being the immediate predecessor $\left(\mathrm{u} \prec{ }^{*} \mathrm{v}\right)$, or their converses. ${ }^{129}$ There is a derived metathety relation of y being between two points $\mathrm{x}$ and $\mathrm{z}$ that holds if either $\mathrm{x} \prec \mathrm{y} \prec \mathrm{z}$ or $\mathrm{z} \prec \mathrm{y} \prec \mathrm{x}$. If $\mathrm{u} \prec$ $\mathrm{v}$ then $\mathrm{u}$ is said to be an ancestor of $\mathrm{v}$, if $\mathrm{u} \prec^{*} \mathrm{v}$ then $\mathrm{u}$ is said to be a parent of $\mathrm{v}$. The structure is just that of a partial ordering and one important problem was that of explaining how a partial ordering can come to approximate a differentiable manifold with a general relativistic metric. Sorkin's solution is to note that in the context of quantum theory we require a probability distribution over the many ways the aether (he would call it that) could be. And there is a very natural way of associating a manifold equipped with a general relativistic metric with such a probability distribution, namely sprinkling - the result of a random selection of at most countably many points from the manifold. ${ }^{130}$

129 In this context 'absolute priority' is appropriate in place of 'frameindependent priority', because we are positing a fundamental relation between regions.

If $\mathrm{u} \prec \mathrm{v}$, then the absolute temporal distance between $\mathrm{u}$ and $\mathrm{v}$ is defined as the length of the longest ordered chain connecting $u$ to $v$, i.e. the greatest integer $n$ for which there are points $u_{0}, \ldots, u_{n}$, such that $u=u_{0}, u_{j} \prec^{*} u_{j}+1$ and $u_{n}=v$. If the absolute temporal distance between $u$ and $v$ is large, then 
I want to emphasise that Causal Set Theory is just the sort of account of the aether that we should hope for a priori. It combines my initial favourite account of the aether with a single primitive relation. The title 'Causal Set theory' suggests that the relation is causation. But that is not essential. Instead I call the relation that of absolute priority.

Causal Set theory does not posit any group theoretic symmetry for the aether. One problem, therefore, is that the Einsteinian (i.e. general relativistic) manifold, which I think of as Space-time, must be derived from the structure of the aether points. Sorkin hypothesises that the probability distribution over the states of a causal set made up of points is given by sprinkling, that is a random choice of representing points from the general relativistic manifold (Sorkin 2003: 9-10). The problem is that of inverting this procedure: given a range of physically possible causal sets, find an Einsteinian manifold such that they are the likely result of sprinklings. Now Sorkin suggests two constraints on the dynamics of causal sets (Sorkin 2003: 13). The first is 'discrete general covariance', which in effect says that the order in which points are born, that is come into existence, is not relevant to the dynamics. Sorkin's second constraint is 'Bell causality', which 'is meant to capture the intuition that a birth taking place in one region of the cause cannot be influenced by other births that occur in regions space-like to the first region' (Sorkin, 2003: 13).

These constraints lead to a formula for the probability of the birth of a new point with specified numbers $\varpi$ of ancestors and $m$ of parents (Sorkin, 2003: 13-14). This probability is proportional to $\lambda(\varpi, \mathrm{m})=$ $\left.\sum \mathrm{t}_{\mathrm{k}} \times(\varpi-\mathrm{m}) ! /(\mathrm{k}-\mathrm{m}) ! \times(\varpi-\mathrm{k}) !\right)$, where the constants $\mathrm{t}_{\mathrm{k}}$ have yet to be specified, and the sum is over all $\mathrm{k}$ from $\mathrm{m}$ to $\varpi$.

A different approach to the dynamics of causal sets is to specify the action associated with a given subset $\mathrm{X}$ of the causal set and rely on the Feynman's sum over histories method of associating probability distributions with actions. Thus in 4 dimensions, the Benincasa-Dowker action associated with $\mathrm{X}$ is the quantity: $\mathrm{S}(\mathrm{X})=\mathrm{N}-\mathrm{N}_{1}+9 \mathrm{~N}_{2}-16 \mathrm{~N}_{3}+$ $8 \mathrm{~N} 4$. Here $\mathrm{N}$ is the number of elements in $\mathrm{X}, \mathrm{N}_{1}$ is the number of chains

sum of all the points $\mathrm{z}$ such that $\mathrm{u} \prec \mathrm{z} \prec \mathrm{v}$ should be a very good approximation to an Alexandrov interval with vertices $u$ and $v$. 
of length 2 in $X, N_{2}$ the number of chains of length 3 in $X$, and so on. (A chain of length 2 is a pair of points $u$ and $v$ such that $u \prec^{*} v$, a chain of length $\mathrm{n}+1$ is $\mathrm{n}+1$ points $\mathrm{u}, \mathrm{w} 1, \ldots, \mathrm{wn}$ such that $\mathrm{u} \prec^{*} \mathrm{w} 1$ and $\mathrm{w} 1, \ldots$ $\mathrm{w}_{\mathrm{n}}$ is a chain of length $\mathrm{n}$. )

Given this, or some other formula, the Feynman method for assigning probabilities given an action, will then specify a probability distribution for a range of causal sets, one that will given some boundary conditions hopefully specify a unique Einsteinian manifold from which that distribution could be arrived at by sprinkling (Surya, 2011: 14). ${ }^{131}$

Thus we may hope to provide the Hauptvermutung (Sorkin, 2003: 10), showing that sprinkling over different Einsteinian manifolds is likely to give different causal sets. In that case we could say that the Einsteinian manifold specified is Space-time, in which the points are located.

Given this Hauptvermutung, causal set theory would specify a differentiable manifold thus replicating one of the major advantages of granulated aether. Moreover if the dynamics really does show that the Einsteinian manifold is specified by the boundary conditions then the Hole Problem is solved.

There is, however, a further problem, noted by Sumati Surya. We would expect the theory of gravity to be compatible with nearly empty Space-time equipped with almost the special relativistic metric. In that, and many other cases, each point is likely to have an infinity of 'parents', in which case the formulae fail to make sense. Or so it might seem, but before judging this I shall state the non-locality problem for Point Discretion starting with the straightforward incompatibility of temporal locality with discrete Lorentz invariance.

\section{The non-locality of discrete Special Relativity}

I begin by considering the non-actual and perhaps impossible case of a discrete analog of Minkowski Space-time. This raises a serious problem

131 Boundary conditions presumably specify the whole of history prior to the points of interest. Contrast this with the continuous case where we only need specify conditions on a suitable hypersurface. 
in the case of the possible and perhaps actual case of an approximation.

To obtain a discrete analog of Minkowski Space-time we represent points by quadruples of integers. Likewise there is a discrete analog of spatial three dimensional Euclidean space with points represented by triples of integers. In the discrete Euclidean case the angle of a rotation is restricted to multiples of right angle, with the axes of rotation restricted to one of the three coordinate axes. This is a version of Weyl's problem. It is noteworthy, therefore, that in the case of the 4 dimensional discrete (analog of) Minkowski Space-time there is a countable infinity of transformations that keep fixed some past light cone $u$. These transformations form the group of all the Lorentz transformations whose matrices have integer values, and whose inverses also have integer values. Moreover, there are enough such Lorentz transformations to establish approximate spatial isotropy, where the approximation may be as accurate as we please. $^{132}$

The point $\mathrm{p}$ represented by a quadruple of integers $<\mathrm{t}, \mathrm{x}, \mathrm{y}, \mathrm{z}>$ may instead be represented by the $2 \times 2$ complex valued matrix $M(p)=[t+z, y+i x] \&[y-$ $\mathrm{ix}, \mathrm{t}-\mathrm{z}$ ]. (I shall use the convention that $\mathrm{n}$ by $\mathrm{n}$ matrix is to be specified by listing the rows in order joined by ampersands.) Given that representation, a Lorentz transformation may be assigned to any $2 \times 2$ complex matrix A of determinant 1, where if the Lorentz transformation maps $\mathrm{p}$ to $\mathrm{q}, \mathrm{M}(\mathrm{q})=$ $\operatorname{AM}(p) A^{*}$. If $A$ is $[a, b] \&[c, d]$ then $A^{*}$ is $\left[a^{*}, c^{*}\right] \&\left[b^{*}, d^{*}\right]$, where $z^{*}$ is the complex conjugate of $\mathrm{z}$. Notice that $\mathrm{A}$ and $-\mathrm{A}$ correspond to the same Lorentz transformation.

We now restrict attention to Lorentz transformations for which the all of $\mathrm{a}, \mathrm{b}, \mathrm{c}$ and $\mathrm{d}$ are Gaussian integers (i.e. complex numbers of the form $\mathrm{m}+$ in, where $m$ and $n$ are integers) such that $a d-b c=1$, and such that $a a^{*}+b b^{*}+$ $\mathrm{cc}^{*}+\mathrm{dd}^{*}$ is even. Then the corresponding Lorentz transformation maps quadruples of integers to quadruples of integers. The special case in which a, b c and d are all real numbers is noted by Schwarz (1976). A family of such transformations is obtained by taking any two Gaussian integers $\mathrm{f}$ and $\mathrm{g}$ and putting $a=2 f^{2}, b=(2 f g+1), c=(2 f g-1), d=2 g^{2}$.

Now the spatial directions of the vectors represented by the quadruples correspond to points on the sphere, which may themselves be represented as ratios of complex number. The effect on the spatial directions of the transformation represented by $[a, b] \&[b, c]$ is then a transformation of the sphere that sends the ratio $u: v$ to $(a u+b v): c u+d v$. The mathematical theory of 
We have, therefore, an initially satisfactory discrete (analog of) Minkowski Space-time with points represented by quadruples of integers. But it suffers from non-locality. Consider a given reference-frame and a given point of discrete Minkowski Space-time. Then not only does it have infinitely many 'parents', that is immediate predecessors, but these occur arbitrarily far in the past and arbitrarily far away in Space with respect to the frame being considered. If therefore we think of direct causal influences being transferred from the immediate predecessors of a point to that point then the direct causal influences are transferred across arbitrarily large gaps of Space and Time. This is not the non-locality that coherent quantum states display, which implies statistical correlations across Space, nor is it restricted to very small times and distances. It involves causation operating across billions of years and light years.

The problem posed by such non-locality may be illustrated by considering point particles travelling at near the speed of light. Suppose $\mathrm{m}$ and $\mathrm{n}$ are large positive integers with no common factor other than 1 . And suppose that $\mathrm{m}^{2}$ units of length is about a light year. So if the unit is the Planck length, suppose $m$ is of the order of magnitude 1024. Consider a particle with trajectory represented by the set of quadruples $\left\{<\mathrm{k}\left(\mathrm{m}^{2}\right.\right.$ $\left.+\mathrm{n}^{2}\right), \mathrm{k}\left(\mathrm{m}^{2}-\mathrm{n}^{2}\right), 2 \mathrm{kmn}, 0>: \mathrm{k} \in \mathrm{Z}$, where $\mathrm{Z}$ is the set of integers, and the coordinates are for the observer's frame of reference. Although this satisfies the criterion that the trajectory seems macroscopically connected with respect to some frame, relative to the observer's frame the particle only exists about once a year. Or, to take a more extreme case, a particle could have only existed twice since the Big Bang. Moreover these

these Möbius transformations is well known and sometimes discussed when considering how the night sky would look to an astronaut passing Earth at a high relative speed. By taking $\mathrm{ff}^{*}$ and $\mathrm{gg}^{*}$ large enough there is a spatial direction $\zeta$, such that the transformation sends all directions other than those close to the opposite of $\zeta$, to some direction very close to $\zeta$. The direction $\zeta$, is specified by the ratio $\mathrm{f}^{*}: \mathrm{g}^{*}$, and so is easily seen to be arbitrarily close to any specified direction. In the case in which the direction is close to the opposite of $\zeta$, we may of course rotate by $180^{\circ}$ first. This establishes approximate spatial isotropy in the sense that given any direction we may, to as good an approximation as we please, map it to any other direction using a Lorentz transformation with integer values for its matrix. 
would be particles of familiar kinds such as photons or neutrinos. I call this the Problem of Occasional Occurrence. This, together with the more general non-locality is a serious problem for Point Discretion.

One conclusion we might draw is that Point Discretion coheres poorly with the hypothesis that the aether is symmetric. We might, instead, deny that there are point particles. But the problem is quite resilient. For, as I now argue, it holds provided the aether approximates discrete Minkowski. Space-time locally and it holds even if there are no point particles.

The discrete structure is at the Planck scale; the events of familiar subatomic particle interaction and so forth interacting take much longer, say N Planck times. Then to say that direct causation is non-local might be interpreted as saying the gap between cause and effect is of the same order of magnitude, N Planck times. One rationale for that interpretation would be that such a gap allows some later event to prevent the effect in question, an effect that has already been guaranteed by the cause. For example, a gamma ray might be about to generate an electron/positron pair when it is hit by some other electron - out of left field, as it were, knocking the gamma ray sideways. It is too late for the electron/positron pair not to come into existence - they have been directly caused - but the gamma ray is now in the wrong place. ${ }^{133}$ This problem is very like the problem faced by proposed examples of backwards causation. If the cause guarantees the effect but comes after the effect, something might prevent the cause even after the effect has occurred.

For this reason, or merely because it is intuitive, I say that there is problematic non-locality if the direct cause precedes the effect by a gap of at least the time the cause (or the effect) takes, namely N Planck times. I now argue as follows. Suppose the cause takes N Planck times and then, one Planck time later, the effect begins. Now consider the same kind of process but occurring in a frame that is moving at near the speed of light relative to ours. Then the gap of 1 unit can be stretched to $\mathrm{N}$ units, although the $\mathrm{N}$ units will then be stretched to $\mathrm{N} 2$ units. With a

By making the interfering particle travel fast enough it could intervene even if the gap is rather less than $\mathrm{N}$ Planck times, but for simplicity I ignore this and consider a gap of $\mathrm{N}$ Planck times. 
gap of $\mathrm{N}$ units we have the problematic non-locality. The only way this argument can fail is if there is no region of Space-time of the required extent for which the Space-time is approximately Minkowskian. A Planck time is of the order of magnitude of $10^{-44} \mathrm{sec}$. We may take the quark scale to be of the order of $10-18 \mathrm{~cm}$, so events at that scale should be of order of magnitude about 10-30 second, making $\mathrm{N}$ of the order of magnitude 1014. But even if $\mathrm{N}$ was as great as 1025, N2 Planck times is about $106 \mathrm{sec}$, which is less than a year and it is plausible that there are regions of about a light year across lasting a year in which the General Relativistic Space-time is a good approximation to Minkowski Spacetime. So a process occurring in a frame of reference moving with respect to ours with velocity very near that of the speed of light will leave a gap long enough for a process taking the same proper time but in our frame of reference to interfere in what is being proposed as direct causation.

Causal set theorists such as Sorkin recognise the non-locality and the consequent need to introduce a cut-off. What this amounts to is the denial that the discrete aether is fully Lorentz invariant because frames of reference with velocities close to the speed of light are prohibited. Note that this cut-off is more severe than the plausible exclusion of frames of reference with respect to which the Big Bang is less than a Planck time ago. For it also excludes the frames with respect to which the Big Bang is many orders of magnitude times 10 billion years ago.

This problem is quite general for Point Discretion, but it is even worse for Causal Set theory with a dynamics that counts immediate predecessors, where we obtain infinite quantities. Maybe this special problem for Causal Set theory can be solved by requiring that no point has infinitely many predecessors, thus excluding as impossible the case of almost flat discrete aether that has always existed. In that case we may hope that a suitable formula for the action will provide us with the Hauptvermutung. Nonetheless the counter-intuitive non-locality remains. So some more drastic solution is required.

I now ask why Granulated Aether is not itself beset by the nonlocality problem, and the answer is that it would be if it were strictly frame-invariant. For consider a granule represented by a pentatope of coordinate quadruples whose base is a spatial tetrahedron. The granule's 
shape is purely topological in that it can as faithfully be represented by any pentatope, whatever the shape. Hence with respect to the 'wrong' frame of reference its immediate predecessor would be years ago. The solution to the problem in this case is to note that the fictitious Spacetime in which the granules are located, and whose coordinates may be used to represent the aether, may be taken to be one in which no granule is located in pentatopes that depart too far from regularity. This fictitious Space-time is not Lorentz invariant and has imprecise simultaneity, implying an imprecise cut-off to the velocities of frames of reference. That is, there might be many permitted frames of reference, namely those with respect to which the granules are not too far from regular pentatopes. None of the frames are ones with respect to which some immediate predecessor of a granule is a long time ago.

A necessary and precise absolute simultaneity is considered problematic because it makes it mysterious why the laws of nature are such that in the idealised case of Special Relativity they are independent of the choice of frame of reference. Neo-Lorentzians such as Craig (2001) accept this mystery as the price they think they must pay for dynamic theories of Time. Assuming they are wrong (Forrest 2008 ) we must avoid this mystery. To do so it suffices that there is a range of permissible frames of reference. If we fix one of them, $F_{0}$, then the range will be specified by a set $\mathrm{S}$ of Lorentz transformations as the set of frames mapped from $\mathrm{F}_{0}$ by some $\mathrm{g} \in \mathrm{S}$. Provided S generates all Lorentz transformations (i.e. provided there is no proper subgroup of the Lorentz transformations containing S) any law of nature that has the same statement in every permissible frame must have the same statement in every frame, as required for Special Relativity.

It should be noted that because the Lorentz group is not commutative there is no principled way of picking out the centre of the range of permissible frames of reference. If there were it could be argued that the central frame was in fact that of absolute simultaneity, re-introducing the problem that I claim to have solved.

This solution to the non-locality problem arises in a natural way in the case of Granulated Aether, but would be the result of an arbitrary cut-off to frames of reference in the case of Point Discretion. This dif- 
ference is the reason why I prefer Granulated Aether (and, in particular, Pseudo-set Granules) to the initially more attractive Point Discretion. I note that Causal Set theory may easily be re-interpreted as a theory of granulated aether. We just replace points by extended simples and take the immediate 'causal' priority of $\mathrm{u}$ over $\mathrm{v}, \mathrm{u} \prec^{*} \mathrm{v}$, as one of three ways in which $u$ and $v$ can share a hyperface. A happy consequence of this reinterpretation of Causal Set Theory is that the number of immediate predecessors may be taken to be finite, as required, for instance, in the Benincasa-Dowker action.

Alternatively, we might resort to Dynamic Triangulation theory, which seeks to develop quantum gravity by using the ideas of classical statistical mechanics but applying them to the division of the aether into cells, which I take to be granules (Ambjørn, Carfora and Marzuoli, 1997). As I understand it, Dynamical Triangulation was initially stated in terms of the length of edges, but it is hoped that a purely topological, that is, qualitative, theory, can be developed. (Ambjørn, Carfora and Marzuoli, 1997: 297-8). The resulting theory will be similar to Causal Set theory.

\section{From discrete aether to geometrodynamics}

Geometrodynamics is the ambitious project of using quantum gravity to provide a 'theory of everything'. In the continuous case it would require that fundamental particles (quarks and leptons) be suitable knots or other topological features in the aether. In the discrete case these particles could be patterns analysable in terms of adjacency, including its special case of immediate causal priority. I would like discrete geometrodynamics to be correct, and it would be a triumph for ontological simplicity, especially on the Pseudo-set Granules version.. But I judge it to be bold in the Popperian sense, and so unlikely prior to testing. ${ }^{134}$ Readers may

134 The noteworthy feature of a Popperian bold conjecture is that prior to testing it is judged improbable that the test will be in conformity with the conjecture. From that it follows both that the conjecture is improbable prior to testing and that if tested and not found wanted it will be significantly confirmed. I noted previously that bold conjectures have been confirmed more often than 
disagree: in the words of an excellent metaphysician, 'I would think the less of reality if it were not so'. ${ }^{135}$ So I do not rely on my judgement in this case. What I shall argue, though, is that discrete theories of the aether are committed to geometrodynamics.

To argue this, consider again the topic of quantum gravity. Some readers may resist the need to quantise gravity. ${ }^{136}$ But in that case we have no reason to believe in discrete aether since, prior to quantisation, General Relativity and its variants are all continuous theories. So the thesis that aether is discrete is committed to the quantum gravity project. Next suppose we undertake this project by treating gravity as somewhat like the other forces and so to be quantised in the same way, using Feynman's sum over histories method or something similar. Initially that would seem to require the aether to be flat or to have some other symmetric shape. For the interpretation of General Relativity as showing that the aether is itself curved in an irregular way, seems to imply that gravity is quite different in origin from other forces. But the initial inference overlooks the possibility of treating all the forces as due to the curvature of the aether, as in the geometrodynamic program. There are, then, two currently feasible methods of treating gravity as like other forces: assimilate gravity to them or them to gravity. It is hard to assign a probability to the third way, namely something quite novel - neither curvature nor forces - that explains both of them. Only if neither of the currently feasible methods succeeds we would seek the novel third way. Given that, as I shall argue, it is far from bold to treat gravity as much like another force, I judge it fairly unlikely that some as yet un-thought-of third way will be required.

we would have expected a priori, and so we should be somewhat less reluctant to affirm them.

135 Said only half in jest by Keith Campbell in conversation.

136 Suppose, for instance, we interpret quantum theory in terms of many worlds in each of which there are particles interacting with each other. Then General Relativity without any quantisation may be interpreted as showing that the worlds are curved by the particles and that between interactions the particles follow geodesics. The case for quantising gravity has, however, been ably made by Thomas Thiemann (2003: 6-7). 
We are left, then, with geometrodynamics on the one hand and gravity-as-a-force on the other. It remains to sketch gravity-as-a force, and to explain why it is unlikely given discrete theory. The sketch will enable me to comment on the differences between gravity and other forces and so reply to the objection that these differences are best understood by taking Space-time to be contingently curved.

Gravity is not exactly like other forces. The first, but perhaps least important difference is the curious combination of (1) the way gravity is characterised by the general relativistic 'metric', but (2) there is still a metric in the case in which we ignore gravity and Space-time is Minkowskian. We might perhaps take this to show that the gravitational field is the deviation from the 'metric' of Minkowski Space-time. But that would be to introduce a necessary structure consisting of the light cones or some equivalent way of characterising Minkowski Space-time, even though it is redundant because there is a contingent gravitational field that replaces the Minkowski structure in all but the matter-free case.

Instead I submit that the matter-free case is not gravity-free, and that the so-called absence of gravity is just the case of an especially straightforward 'metric', that of Minkowski Space-time. Additional support for this simplification may be derived from the probable need to introduce a cosmological constant, which also shows that the matter-free case is not free of all gravity-like features..

It is initially probable, therefore, that the necessary structure of the aether does not include the light cones or, equivalently, the partial ordering of absolute priority. This coheres with the case, made in the previous chapter for decoupling the metric and order aspects of Space-time, because neither the aether nor Space-time is ordered by anything necessary but rather by the contingent gravitational field. In the next chapter I shall assume that affine aether has no necessary light cone structure, even if necessarily it has some light cone structure. The alternative, in which light cones are necessary presents no difficulties and, in the point-free case, is less complicated, which should occasion some doubts as to whether the cone-free approach is correct. It will also turn out that in the case in which the structure of the aether is not affine but that of de Sitter Space-time the cosmological constant implies a light cone structure. 
The most straightforward case, however, is that in which affine geometry holds of the aether. This is the geometry in which there are hyperplanes and an equivalence relation on hyperplanes of being either identical or non-identical but parallel, where parallel implies nonidentical. This equivalence relation is such that such the analog of that Euclid's Fifth Postulate holds, namely parallel hyperplanes never intersect. Affine geometry may be characterised axiomatically using the primitive relation of metathety, namely the three-place relation between points $\mathrm{p}, \mathrm{q}$, and $\mathrm{r}$ that holds just in case $\mathrm{p}, \mathrm{q}$, and $\mathrm{r}$ lie on some line with $\mathrm{q}$ between $p$ and r. (Coppel 1998). A less fundamental description is as the geometry in which Space-time is represented by quadruples of real numbers in such a way that hyperplanes correspond to linear equations, but without any metric structure or specification of perpendiculars. So, for instance, although hyper-ellipsoids may be characterised there are no special hyper-ellipsoids designated as hyperspheres.

The second difference between gravity and other forces may be illustrated by considering the way a field acts on a single particle - a special case that generalises to many particles and to the way the field acts on itself. The dynamics is constrained by the action, which, taking Planck's constant to be $2 \pi$, is $\int p_{q}(d q)$, where: (1) the integral is over the path of the particle, and (2) $\mathrm{p}_{\mathrm{q}}$ is a real valued linear function on the vector space $\mathrm{T}$ of translations of Space-time (displacement vectors) ${ }^{137}$. The linear real valued functions on $\mathrm{T}$ form a vector space, $\mathrm{T}^{*}$, the dual of $\mathrm{T}$. The vector $\mathrm{p}_{\mathrm{q}}$ itself depends on the position $\mathrm{q}$. and because we put Planck's constant equal to $2 \pi$, may be identified with the energymomentum vector at $\mathrm{q} .{ }^{138}$ The energy-momentum is a property of the

137 This generalises: (1) we usually consider either a field or matter that is spread out, in both cases the energy-momentum vector is replaced by a tensor; and (2) in the curved case $\mathrm{T}$ is the tangent space at $\mathrm{q}$.

138 Strictly speaking, the energy-momentum should be thought of as belonging to an affine not a vector space. The difference is that a vector space is an affine space with a distinguished member, the zero vector. In the case we are considering the choice of a zero vector introduces gauge invariance, which shows we should not treat $\mathrm{p}_{\mathrm{q}}$ as a member of a vector space. The gauge in- 
particle and so not defined for all locations but merely those on the path, and it will vary as the particle is affected by the field.

The field affects the motion of the particle by specifying how the energy-momentum varies along the path. Such a path-dependent correlation is an affine connection in the differential geometers' sense. For every point $q$ of Space-time and every vector $u$ in $T$ the connection specifies a way of correlating energy-momenta at $q$ with energy-momenta at a point $\mathrm{q}+\mathrm{u}$, displaced by $\mathrm{u}$ from $\mathrm{q}$, for a particle travelling in a straight line. So the connection that characterises a field is a mapping assigning to each position $q$ and each vector $u$ in $T$ a mapping $F$ that correlates the energy-momenta at $\mathrm{q}$ with those at $\mathrm{q}+\mathrm{u}$. The mapping $\mathrm{F}$ preserves the affine structure and therefore given an arbitrary choice of zero energymomentum at each point it may be analysed as the composite of two mappings, a translation and a linear transformation that leaves the zero vector fixed. We may then treat the translation as the non-gravitational aspect of the field and the linear transformation as the gravitational field itself. $^{139}$

In a unified field theory we should expect there to be a single field but it could still have these two components, so in that sense gravity would be distinct from the rest of the field. ${ }^{140}$

variance in question is shown by considering a smooth function $r$ assigning a member of $\mathrm{T}^{*}$ to every location and whose exterior derivative is zero. If we replace $\mathrm{p}_{\mathrm{q}}$ by $\mathrm{p}_{\mathrm{q}}+\mathrm{r}(\mathrm{q})$ that has no effect on the difference between action integrals joining two given points and hence no effect on the dynamics.

I understand that Einstein rejected this way of unifying fields. (He was considering only the electromagnetic and gravitational field.) His rejection seems to have been because he tried to unify the fields by generalising General Relativity, relying on the curvature to specify the evolution of the unified field.

In a unified theory theenergy- momentum space could be a larger space and perhaps not even affine. (The unified theory is even more unified if the symmetry group of the momentum space is a 'simple' group, that is, one without a normal subgroup.) In any case, we generalise from energymomentum vectors to energy-momentum tensors. Then the translation adds to the angular momentum in a way that is said to be gravity acting on the in- 
There is one additional feature of the dynamics that might or might not be fundamental, but is worth mentioning, to counter readers' worries that I have not interpreted General Relativity as they know it. Energymomentum space is not merely affine, it has, as far as we know, a necessary Minkowski-space structure, with a constant $\mu$, characteristic of the kind of particle, which is usually taken to be positive and to be the square of the rest mass. If the energy-momentum is represented by the quadruple $<\mathrm{e}, \mathrm{p} 1, \mathrm{p} 2, \mathrm{p}_{3}>$ then $\mu=\mathrm{e}^{2}-\mathrm{p}_{1}{ }^{2}-\mathrm{p}_{2}{ }^{2}-\mathrm{p}_{3}^{2}$. Because the connection preserves not merely the affine structure of the momenta but $\mu$ is preserved, the connection assigns a member of the Poincare' group (a combination of a translation and a Lorentz transformation) to each point $\mathrm{q}$ and vector $\mathrm{u} .{ }^{141}$ Strictly speaking it is a Minkowski connection, therefore.

This brings me to the apparent superiority of the curved Space-time interpretation of General Relativity, namely the way in which curvature enters into the equations. My response is that curvature does not need to be understood in terms of a 'metric', which is a natural way of thinking only if we are already thinking of a 'metric' in the flat case, as with Minkowski Space. Instead, therefore, I note that the idea of curvature arises whenever we have an affine connection, and a 'metric', if there is one, defines the curvature by defining an affine connection. The translation component of the connection does not affect the curvature, hence the link between curvature and gravity. ${ }^{142}$

I anticipate the objection that the connection makes the affine or other symmetric structure redundant and a differential manifold would do instead. My response is that the affine or other symmetric structure of the aether enables us to preserve Newton's First Law, with friendly

trinsic angular momentum, the spin. This appears to be the motivation for modification of General Relativity by Élie Cartan (1922.)

141 Because the standard case is one in which the underlying Space-time is curved it would be usual to describe the connection as an assignment of a member of the Lie algebra of the Poincaré group to $\mathrm{q}$ and a tangent vector. 
amendments. When the gravitational influence of other matter is negligible and when matter and the non-gravitational forces are also negligible the trajectory of a particle differs negligibly from a straight line (or analog).

Without in any way minimising the mathematical work required, I judge, therefore, that there is only one potential obstacle to a unification of forces on the assumption of a background symmetric Space-time, such as affine space. This is the notorious problem of the divergences. When we use the Feynman sum-over-histories method of quantising a system we find that all the processes we expect to occur have exactly similar duplicates in frames travelling with high velocities relative to us. Unfortunately the appropriate 'probability' measure on the set of frames of reference is the unique (up to a multiplicative constant) Lorentz invariant one, and this is not a probability measure, with total value 1, but has infinite total value. This is, I claim, the ultimate source of the divergences, which may be avoided by means of range of permissible frames of reference, as used in the case of Granulated Aether. That amounts to both an ultraviolet cut-off (excluding frames moving fast towards us) and an infrared cut-off (excluding frames moving fast away). We need to avoid ad hoc choices of the range of privileged frames, but fortunately there is an obvious source of cut-offs, the postulate that the some fundamental fermions of non-zero rest mass have extended locations. For the sake of being definite, suppose an electron has some (noninfinitesimal) positive diameter when it is in a rest frame. Then if it is travelling very close to the speed of light with respect to us its diameter at right angles to its velocity will too large to avoid other electrons that are travelling at low speeds. The postulate of extended electrons puts two constraints on the dynamics, the first is to put an upper limit on the number of them per unit volume and the second is to put an upper limit on their relative velocities. This supposes that no two electrons intersect, but that is plausible since they are fermions.

This way of avoiding the divergences is not ad hoc because there are two reasons why point particles are improbable. The first, which is so elementary it makes me blush, is that point particles moving in straight lines have an infinitesimal probability of collision, and so the attractive idea that particles interact by transferring energy-momentum 
(and spin) on contact requires them to be extended. The second is that I am taking the aether to be the one fundamental substance and hence particles are just regions of aether with suitable properties. Combining this with the superiority of point-free Arntzenius Continuum over an aether composed of points I conclude that there are no point particles.

It only remains to show that symmetric Space-time is improbable given granulated aether. That follows from the way the aether is more fundamental than Space-time. It is not as if we start with some intuition that Space-time is flat and then cut it up into pentatopic domains that we declare correspond to the granules. Instead we start with what is fundamental, the granules and then ask why they should fit together in just such away that the Space-time they are located in is flat, or otherwise symmetric. If we have no answer to that question then we should suppose it is improbable, and that the arrangement of granules is higgledypiggledy but such that a fictitious Space-time in which they are located is flat enough at anthropocentric scales (neither too small nor too large).

This completes my case for the disjunction: either granulated aether without symmetric Space-time but with geometrodynamics, or symmetric Arntzenius Continuum, with gravity being treated much like the other forces. I shall conclude this chapter by considering some further arguments for discrete aether. Regrettably none of them succeed.

\section{The case for discrete time}

Why does the aether persist? Perhaps the most intuitive answer is that every part of the aether endures forever, that is it lacks any division into temporal parts. But I have two reasons for rejecting that thesis. The first is that it implies an aether current, something that Michelson and Morley looked for, and which we might expect to find some empirical evidence for if it occurred, but we do not. The second is Lewis' problem of temporary intrinsics (1986: 202-5): the very same portion of the aether would have to have different intrinsic properties at different times, contrary to the Indiscernibility of Identicals. ${ }^{143}$

143 For a survey of solutions to the problem of temporary intrinsics see (Gallois 2011). One is to say that persisting entities have time-dependent properties, another that they time-dependently instantiate properties. If either of these 
We require a different hypothesis to explain the universe's persistence. The most straightforward is that each stage is directly caused by some of the earlier stages (Hans Reichenbach's genidentity, 1957: 270271). Here I say that $u$ directly causes $w$ if that causal relation does not depend on u's causing $\mathrm{v}$ that causes $\mathrm{w}$, for some $\mathrm{v} .{ }^{144}$ If persistence is due to direct causation, then causation, at least as it applies to the persistence of the aether, is discrete, in the sense that indirect causation always depends on finite causal chains. To be sure, discrete causation does not entail a discrete theory of the aether, but I now argue that the combination of discrete causation with a continuous theory of the aether is awkward.

Suppose the aether is temporally discrete but spatially continuous. Then there cannot be any continuous processes, because the structure would have to change suddenly at the end of a region. So I infer that if the aether is spatially continuous it is also temporally continuous. Combining that with discrete causation requires that a layer of aether divisible into thinner layers be caused as a whole by an earlier layer similarly divisible, with divisions between the layers.

Might not we then have 1 unit thickness followed by $1 / 2$ followed by $1 / 4$ etc Zeno-style, resulting in an explanation of persistence for only 2 units of time in all? To exclude this, we have to posit that layers of some fixed thickness, say the Planck time, directly cause later layers of fixed thickness. The objection to this is that unless Time is discrete the uniform thickness of the layers is an ad hoc hypothesis to save discrete causation, whereas discrete causation is implied by a discrete theory of the aether.

holds then there must be a Time distinct from the aether that fills Space-time. Time thus distinguished from Space-time is considered below, when discrete Time is discussed. Another solution is the presentist insistence that only the present is real. I fail to see how the persistence of the aether or any other physical thing can be explained by presentists without invoking either an enduring or timeless sustaining cause, something I also consider below. cluded is that u's causing $\mathrm{w}$ depends on the intermediate causation. 
That was the argument and I now consider alternative explanations of persistence of the aether. Some would say, 'It just does'. I am not impressed. For there are answers to the question and so not to answer it is to multiply mysteries.

Do laws of nature provide an explanation of the persistence of the aether? I have not, and shall not, assume that causation is primitive, so direct causation might well be itself explained by laws of nature - conservation laws maybe. But to provide an alternative we require laws that explain why the aether persists even if not all causation depends on direct causation, or perhaps even if there is no causation at all. First suppose the law states that if the aether exists at time $t$ it exists at some later time. In that case, we have the Zeno-style problem that the aether persists for 1 second then $1 / 2$ second then $1 / 4$ second etc and we have not explained how it persists for 2 seconds. Next suppose the law states if the aether exists at time $t$ then it exists at time $t+\varepsilon$. Then rather than being an alternative to discrete causation it implies discrete causation. For if we consider the slice of aether that has existed for the last $\varepsilon$ it directly causes the slice existing for the next $\varepsilon$. So it is not that easy to state how the law works. My suggestion is that it would have two parts. The first, a metric completion law, stating that if there is a sequence of time coordinates, $\mathrm{t}_{1}, \mathrm{t}_{2}$, etc at which the aether exists and this sequence converges to a limit, $t^{*}$, then the aether exists at $t^{*}$. The other would be the law that if the aether exists at time $t$ it exists at some later time. Together these laws imply that the aether exists forever.

I have two objections to these or to any other laws that imply the continued existence of the aether. The first is that they work too well; implying that necessarily if the aether ever exists then it exists forever. I incline towards the position that the aether always has and always will exist but this does not seem to be necessary. For if the universe were expanding somewhat less rapidly it would collapse into a final crunch. My other objection is audience-specific and directed at those who assume General Relativity in a version that requires all laws to be covariant. It is that unless Time is discrete, the time coordinate may be re-scaled so that the infinite future becomes finite. So the law in question would not satisfy the general relativistic requirement of covariance. By contrast, the ex- 
planation of persistence in terms of discrete causation is covariant. If we re-scale Time we merely change the shape and size of the layers or cells of aether without affecting the causal description.

An alternative explanation that should be taken seriously is that there is an enduring entity, such as the God of classical theism, that sustains the aether. So the aether is not caused by earlier stages. Or, the divine persistence might be due to each divine stage directly causing the next, but this persisting God could then sustain the aether.

My challenge, then, is directed at those, whether theist or atheist, who reject the idea that the aether is sustained by God, to explain why it persists without relying upon discrete aether.

That was the challenge. I now take it up by distinguishing between the Space-time in which the aether is located and the temporal order. I do so in the context of the Growing Block theory, with the block in question being the aether. That is, I suppose the grammatical present is pre-tensed and if we want to restrict attention to what exists now we have to be explicit and use the word 'now' or something equivalent. So, as a Growing Block theorist I say that the aether that exists at some time in the past is a proper part of the aether that now exists. I shall also assume that Space-time is symmetric, because that is what I believe holds unless the aether is discrete, and I am here considering the case from discrete Time to discrete aether, so I can argue by reductio ad absurdum, supposing the aether is not discrete.

On this Growing Block theory the truth is what is now true, which is not the same as what was true a while ago. A. For some $a$ and $b$, ' $a$ is the aether' is now true but ' $b$ is the aether' was true and $b$ is a proper part of $a$. Space-time does not change: truths about it are true at all times. So the aether grows into Space-time. (I explain in the next chapter how the whole of Space-time can be constructed from the aether that does not fill it.) Space-time has a partial ordering of its points with respect to frame-independent priority. So it is not just a hyperspace, but on the Growing Block there is an additional temporal ordering of the past (and present) locations of the aether, with the locations of earlier ones being included in those of later ones. The passage of Time concerns the growth of the aether with respect to that temporal ordering. Rather than call this temporal ordering hyper-time it would be more appropriate, alt- 
hough pedantic, to re-name Space-time as Space-hypotime. In any case we may distinguish the metric aspects of Time, which are thoroughly unified with those of Space, from the ordinal aspects. ${ }^{145}$ This temporal ordering I take to be a total not merely a partial ordering. This is not the place to defend the resulting theory of Time. Rather I have illustrated how we might distinguish the two aspects of Time. Given that we do, so we may then solve the problem of why the universe persists without invoking God as sustaining cause, by taking the temporal ordering to be discrete in the sense that for every $x$ and $y$ there are only finitely many $z$ after $\mathrm{x}$ and before $\mathrm{y}$. In that case the rate of passage of Time may be measured in seconds, not, note, seconds per second: with the rate being the thickness of the layer between two successive stages of the growth of the aether, which is analogous to tree rings. There is no reason to expect this growth either to be spatially or temporally uniform.

I conclude that unless the aether is sustained in existence by God or some other sustaining cause, Time is discrete, but by distinguishing Time from the hypotime that is the fourth dimension of Space-time, I resist the conclusion that the aether is itself discrete.

Supertask arguments, even if otherwise acceptable, likewise show that it is the temporal ordering that is discrete, rather than directly showing that the aether is. These arguments proceed by showing that if Time is continuous then there is an infinite sequence of events such that any finite number are jointly possible but the whole series is so counterintuitive that we might judge it impossible. Then we have a contradiction, because if the whole series is impossible it must cease to be possible before it is completed and hence some finite number of the events are not jointly possible. There are many ingenious examples but the following variant on the Ross-Littlewood Paradox suffices (van Bendegem, 1994). At time 0, some kind of particle, a magnetic monopole say, is unique. Then some events result first in the creation of two new monopoles and then the destruction of the old. (By 'then' we mean at a later time with respect to some arbitrarily chosen frame of reference.) A short

More generally the ordering is that some enduring and everlasting states of affairs come into existence before others. I am not claiming that all states of affairs are everlasting only the fundamental ones on which others depend. 
time later three new magnetic monopoles are created and then the two already existing are destroyed. By chance this is repeated more and more often so that over a second interval there are more and more, but at the end of the process there are none, because every monopole that came into existence has ceased to exist. The whole process is of infinitesimal probability but seems nonetheless to be possible unless Time is discrete. My response is, 'So what?' Supertask enthusiasts might, however, consider it impossible and hence they have a reductio ad absurdum of the supposition that Time is continuous.

My conclusion, then, is that arguments for the discrete character of Time may well succeed, but if they do the advocate of continuous aether should take this as showing that Time is not the same as the fourth dimension of the Space-time in which continuous aether is located. To naturalists, the idea of Time distinct from Space-time might seem a metaphysical extravagance, but my guess is that the naturalists in question will not be impressed by the argument for discrete Time in the first place. 